\title{
Estimation of Poverty Based on Remote Sensing Image and Convolutional Neural Network
}

\author{
Peng Wu, Yumin Tan \\ School of Transportation Science and Engineering, Beihang University, Beijing, China \\ Email: ZY1713401@buaa.edu.cn, tanym@buaa.edu.cn
}

How to cite this paper: $\mathrm{Wu}, \mathrm{P}$. and Tan, Y.M. (2019) Estimation of Poverty Based on Remote Sensing Image and Convolutional Neural Network. Advances in Remote Sensing, 8, 89-98.

https://doi.org/10.4236/ars.2019.84006

Received: October 24, 2019

Accepted: December 16, 2019

Published: December 19, 2019

Copyright ( 2019 by author(s) and Scientific Research Publishing Inc. This work is licensed under the Creative Commons Attribution International License (CC BY 4.0).

http://creativecommons.org/licenses/by/4.0/

\section{cc) (i) Open Access}

\begin{abstract}
Poverty has always been one of the topics concerned by governments and researchers all over the world, especially in developing countries. Remote sensing image is widely used in poverty estimation because of its large area observation, timeliness and periodicity. In this study, we explore the applicability of convolution neural network (CNN) combined with remote sensing image in regional poverty estimation. In the 2016 economic indicators estimation of Guizhou Province, China, the Pearson coefficient of per capita GDP (PCGDP) reached 0.76 , which means that the image features extracted by CNN can explain the change of PCGDP of county level economic indicators up to $76 \%$. Compared with other methods, our method still has high precision. Based on these results, we found that convolutional neural network combined with remote sensing image can be used in regional poverty estimation.
\end{abstract}

\section{Keywords}

Poverty, Convolution Neural Network, Remote Sensing Image, Economic Indicators, Guizhou, PCGDP

\section{Introduction}

Poverty as a problem has been perplexing governments all over the world, especially developing countries. It is very important for policy makers and researchers to analyze the conditions and causes of poverty, which is helpful to reduce poverty. The traditional way of poverty measurement mainly depends on the ground survey data [1]. However, it takes a long time and is expensive to obtained data [2]. Some countries have not even collected such data [3].

Because remote sensing data can provide large-scale, multi-temporal and spatial resolution surface information, it is widely used in regional poverty estimation. As a new kind of remote sensing data, nighttime light remote sensing data 
records the artificial light in human settlements and is widely used in poverty estimation [4] [5]. Various socio-economic parameters have been estimated by using nighttime light remote sensing data, such as: carbon dioxide $\left(\mathrm{CO}_{2}\right)$ emissions [6] [7], gross domestic product (GDP) [8] [9], population [10] [11], etc. There are two kinds of widely used nighttime light data, which are the nighttime light composite data obtained by the Defense Meteorological Satellite Program's Operational Line scan System (DMSP-OLS) [12] [13] and the new generation nighttime light composite data from the Visible Infrared Imaging Radiometer Suite (VIIRS) Day-Night Band (DNB) carried by the Suomi National Polar-orbiting Partnership (NPP) Satellite [14]. DMSP-OLS data have some shortcomings, such as strong light saturation, coarse spatial resolution and so on [15]. NPP-VIRS data is calibrated to DMSP-OLS Data with better quality. In terms of spatial resolution, 15 arc seconds, 500 meters DMSP-OLS data are better than 30 arc seconds, and 1000 meters NPP-VIRS data can provide more artificial lighting information at night in human settlements [16] [17]. In the use of DMSP-OLS data, Noor et al. [18] used the data to calculate the Pearson coefficient of household asset index; the best result was 0.64, which confirmed the correlation between DMSP-OLS data and socio-economic indicators. Li et al. [19] explored the potential of NPP-VIRS night light images for regional economic modeling in China. Yu et al. [20] used NPP-VIRS data to estimate poverty at the county level in China.

In a recent paper [21], the features extracted from remote sensing images trained by convolution neural networks (CNNs) are used to estimate poverty, which explains up to $75 \%$ of the changes in local economic and living indicators. All the satellite image data used in this study are open and free, which promotes an important step in using such satellite image data to estimate economic indicators without expensive and time-consuming ground statistical surveys. There is no assessment of transfer learning methods to predict changes in economic well-being over time in specific regions. Perez et al. [22] used the Wasserstein generative adversarial networks (WGAN) to construct the semi supervised classification multitask learning to estimate the household asset wealth index (AWI) in Africa. They constructed an end-to-end multitask learning model for a series of classification tasks, including luminous intensity, population density, distance to the nearest road, land cover type and AWI. However, the WAGN model is difficult to train. In the development of convolution neural network, He et al. proposed the deep residual network (ResNet) [23] in 2014, and solved the problem of gradient disappearance caused by increasing depth in the neural network. Lin et al. proposed the feature pyramid network (FPN) [24] in 2017, obtained the feature layers of different resolutions, and improved the detection accuracy of small objects. Based on the development of these technologies [21] [22] [23] [24], we hope to combine ResNet-50 and FPN to build a classification model for training remote sensing images to obtain image features reflecting regional economy. Different characteristic map P2 to P5 of FPN is used to classify four 
kinds of remote sensing data, which are nighttime light data that can reflect the artificial light in human settlements, Normalized Vegetation Index (NDVI) [25] [26] data reflecting regional vegetation conditions, Modified Normalized Difference Water Index (MNDWI) [27] effectively distinguishes Water and urban areas, and Normalized Difference Built Index (NDBI) [28] data reflecting urban and non-urban regional conditions. Different from the previous research [21], [22], we choose ResNet-50 and FPN to build a new convolution neural network model to classify and learn different remote sensing data, so that the convolution neural network model can learn more characteristics that can reflect the changes of regional economy. When the model is trained, the features are extracted for the estimation of economic indicators. The details of the experiment are described in subsequent sections.

\section{Materials}

\subsection{Investigation Area}

Guizhou (Figure 1) is a provincial administrative region of the People's Republic of China. Its capital is Guiyang. It is located in the southwest of China and consists of 88 cities, districts and special zones. The land resources in the study area are mainly mountains and hills, rich in mineral resources, less plains and less cultivated land per capita. Due to historical, geographical, cultural and political factors, the economic level of Guizhou Province has always been at the lowest level in the country. In 2012, the office of the leading group for poverty alleviation and development of the State Council issued a list of 665 key counties for poverty alleviation and development work in China, aiming to lift these counties out of poverty. In 2016, Guizhou Province was composed of nine cities and prefectures: Guiyang, Liupanshui, Zunyi, Anshun, Bijie, Tongren, Qianxinan, Qiandongnan and Qiannan. Among them, there are 66 poor counties, the number of which is far more than that of other regions, with a GDP of 1177.673 billion yuan, ranking 24th in 31 provinces, regions and cities in China [29].

\subsection{Dataset}

In this study, the experimental data include 2016 landsat 8 image, NPP-VIRS nighttime light image and Guizhou annual statistical yearbook data. The landsat 8 image of 88 cities, districts and special zones in Guizhou is generated by Google Earth Engine's Landsat Simple Composite tool. We divide the image of 88 regions into $256 \times 256$ pixels size image tiles. Because the vector boundary of each region is irregular, the whole black image will be removed. Spectral index images were obtained from landsat 8 images collected on the Google Earth Engine, which were similarly divided into image tiles for trained.

The nighttime light data acquired by the Suomi National Polar-orbiting Partnership (NPP) Satellite and two types of NPP-VIRS data are available on the NOAA web site: annual and monthly data. Download 2016 annual nighttime light data from NOAA website and the data is processed and the temporal light 


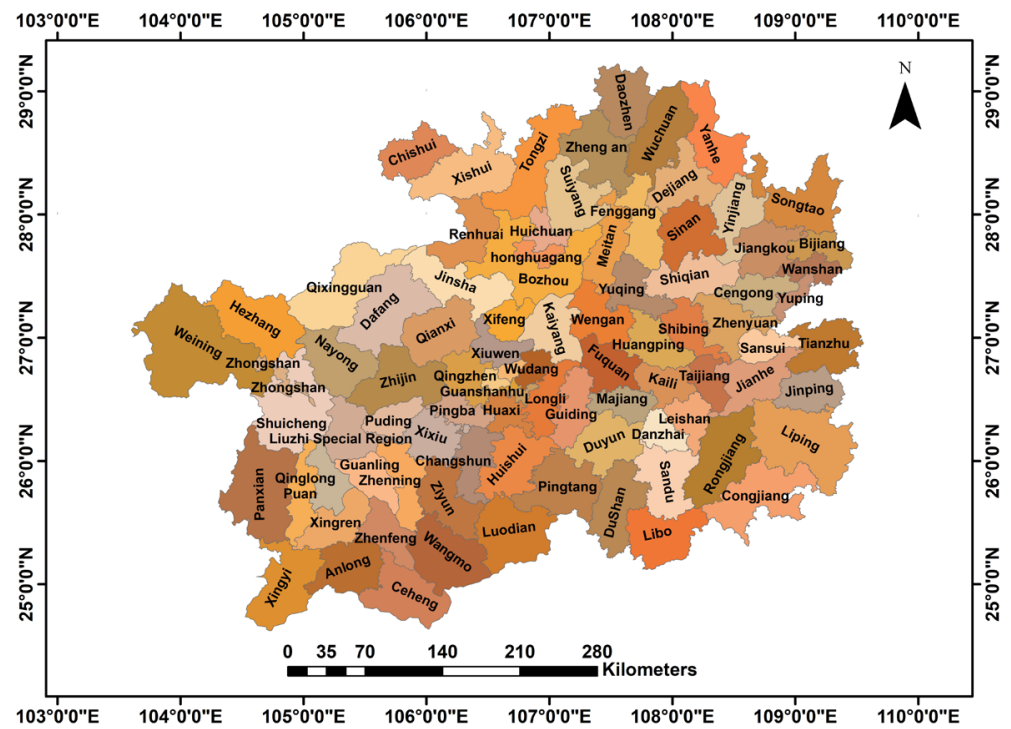

Figure 1. Case study area: Guizhou is composed of 88 cities, districts and special zones. The economic development level of each region is quite different, among which the regional economic development level near the provincial capital city is better than other regions far away from the provincial capital.

and some non-light values are removed. Similarly, the nighttime light images of each city, district and special zone are trained in small tiles, just as we did with landsat 8 satellite images. The 2016 statistical yearbook was downloaded from Guizhou bureau of statistics, in which we collected some economic indicators: Per capita gross domestic product (PCGDP), total retail sales of consumer goods (TRSCG) and general public financial budget revenue (GPFBR). Table 1 shows the data used in the experiment.

\section{Methods}

Our method is to use convolutional neural network $(\mathrm{CNN})$ to extract features from remote sensing images, and then use the extracted features to estimate economic indicators. The specific steps are as follows: First, the deep residual network (ResNet-50) and the feature pyramid network (FPN) are combined to establish the classification model. After the P2-P5 feature map of FPN, a global average pool layer is added, and then a classifier composed of 1024 neurons and a softmax activation layer is constructed to classify the nighttime light data and the spectral index image. Secondly, when the model is trained, the output of the global average pooling layer is extracted as the feature, just like the previous research [22] [30]. Finally, the ridge regression model is constructed to estimate the economic indicators using the features obtained in the previous step and the actual ground economic survey data. It is noted that in ridge regression, the double nested cross validation method is used to estimate the economic indicators, and the inner loop of cross validation is used to find the optimal weight of the regular term (super parameter). This weight is used to predict the economic indicators of the test set in the outer loop of double nested cross validation. The 
Pearson coefficient $\left(\mathrm{R}^{2}\right)$ of the actual economic indicator and the estimated economic indicator is used to evaluate the performance of our method. The overall method flow is shown in Figure 2.

\section{Experimental and Results}

In Section 3, ResNet-50 and FPN are used for classification tasks. The classifiers after P2 feature layer are used for classification of nighttime light intensity. Similarly, the classifiers after P3 to P5 feature layer are used for classification of NDVI, MNDWI and NDBI respectively. The classification categories of four kinds of data are confirmed by Gaussian mixture model. The four data classification categories are shown in Table 2. The input of convolutional neural network is Landsat 8 image and the output is the category of nighttime light intensity (NLI), NDVI, MNDWI and NDBI. Table 3 shows the training accuracy and test accuracy of the four classification tasks. After the training of the model, the output of the global average pooling layer in the convolutional neural network is extracted as the feature, and the 256-dimensional featurevectors of the four classification tasks are combined into a 1024-dimensional featurevectorsas the image features extracted from the remote sensing image by CNN. Finally, we use the economic indicator data from the statistical yearbook and the corresponding image features extracted from the remote sensing image by $\mathrm{CNN}$ to train the ridge regression model to estimate the economic indicator.

In ridge regression model, we use principal component analysis (PCA) to reduce the dimension of features to avoid over fitting, in which 1024-dimensional feature vectors are reduced to 100-dimensional feature vectors. The 10-fold cross-validation method was used to estimate the 2016 economic indicators of Guizhou province, and the process was repeated 20 times. Finally, we took the average value of all $R^{2}$ of 20 times as our final result. In this study, we estimate the three economic indicators of PCGDP, TRSCG and GPFBR. The Pearson coefficients of the three economic indicators are $0.76,0.72$ and 0.65 respectively, as shown in Figure 3, Figure 4 and Figure 5. Compared with the previous experimental results [21] [30], although the estimated economic indicators are different, the Pearson coefficient $\left(R^{2}\right)$ of economic indicators is also in this range, indicating that our method can be applied to the economic indicators estimation of Guizhou Province, and the estimated economic indicators result is reasonable.

In order to test whether our method is better than other methods in the estimation of economic indicators, we also calculate the results of estimating economic indicators directly using the nighttime light (NTL) data. The sum of night light of cities, districts and special zones in Guizhou Province is used to estimate three economic indicators by linear regression model, among which the $\mathrm{R}^{2}$ (Table 4) of PCGDP, TRSCG and GPFBR are $0.31,0.58$ and 0.5 respectively.

\section{Conclusions}

The present study demonstrates that CNN combined with remote sensing image to estimate poverty and identify regional poverty, especially the estimation of 
economic indicators, provides a potentially quick and inexpensive method. These features extracted by deep learning can explain up to $76 \%$ of the variation in local economic outcomes.

Table 1. Dataset characteristics used in this study.

\begin{tabular}{cccc}
\hline Dataset & Spatial resolution & Temporal resolution & Time Line \\
\hline Landsat 8 image & $30 \mathrm{~m}$ & 1 year & 2016 \\
Spectral index image & $30 \mathrm{~m}$ & 1 year & 2016 \\
Nighttime light data & $500 \mathrm{~m}$ & 1 year & 2016 \\
Economic survey data & City, district and special zone & 1 year & 2016 \\
\hline
\end{tabular}

Table 2. Classification category interval of four kinds of data.

\begin{tabular}{ccccc}
\hline Class & NLI & NDVI & MNDWI & NDBI \\
\hline 1 & $0-0.005$ & $-5.62-0.25$ & $-0.38--0.22$ & $-0.39--0.23$ \\
2 & $0.005-0.17$ & $0.25-0.51$ & $-0.22--0.10$ & $-0.23--0.10$ \\
3 & $0.17-33.45$ & $0.51-0.77$ & $-0.10-0.06$ & $-0.10-0.03$ \\
\hline
\end{tabular}

Table 3. Classification category interval of four kinds of data.

\begin{tabular}{ccc}
\hline Data & Training accuracy & Testing accuracy \\
\hline NI & $99.84 \%$ & $90.62 \%$ \\
NDVI & $100 \%$ & $100 \%$ \\
MNDWI & $100 \%$ & $93.75 \%$ \\
NDBI & $100 \%$ & $100 \%$ \\
\hline
\end{tabular}

Table 4. The Pearson coefficient results of the economic indicators estimated by the linear regression model and the actual economic indicators.

\begin{tabular}{cccc}
\hline Method & PCGDP & TRSCG & GPFBR \\
\hline Linear regression model (with NTL data) & 0.31 & 0.58 & 0.5 \\
\hline
\end{tabular}

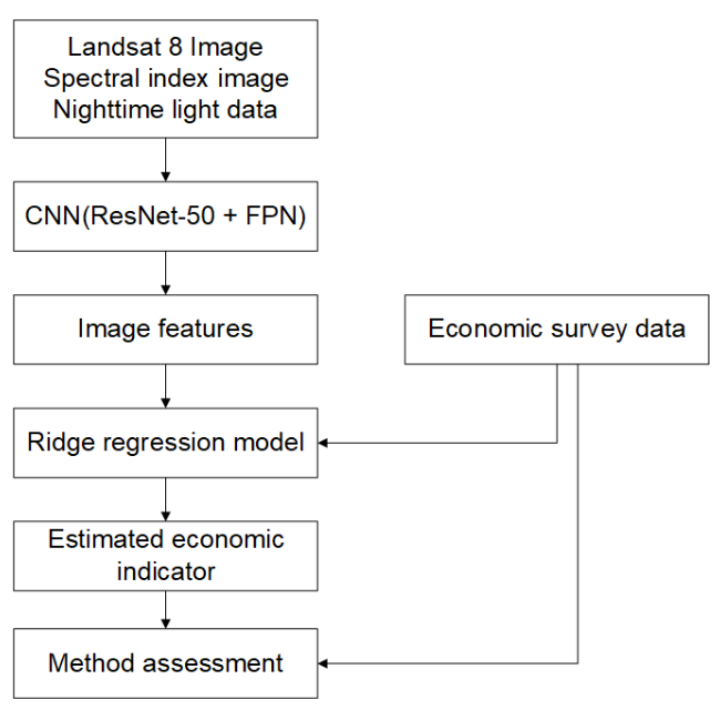

Figure 2. Method flow. 


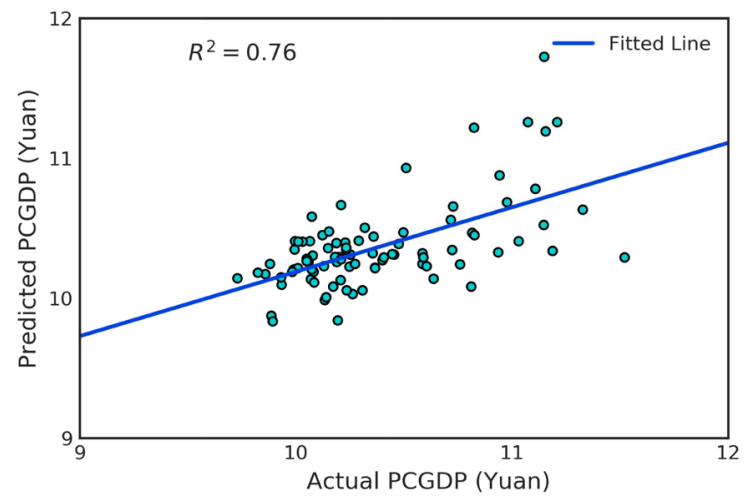

Figure 3. The Pearson coefficient $\left(\mathrm{R}^{2}\right)$ of the predicted PCGDP and the actual PCGDP, in which the blue line is the best fitting line.

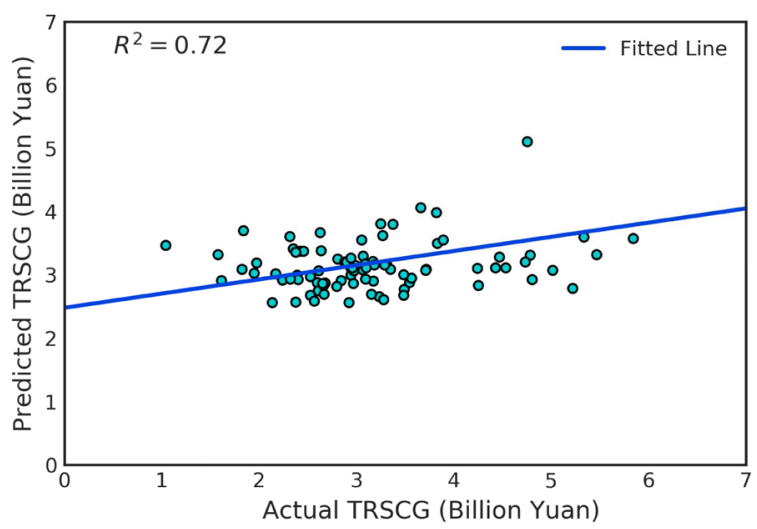

Figure 4. The Pearson coefficient $\left(\mathrm{R}^{2}\right)$ of the predicted TRSCG and the actual TRSCG, in which the blue line is the best fitting line.

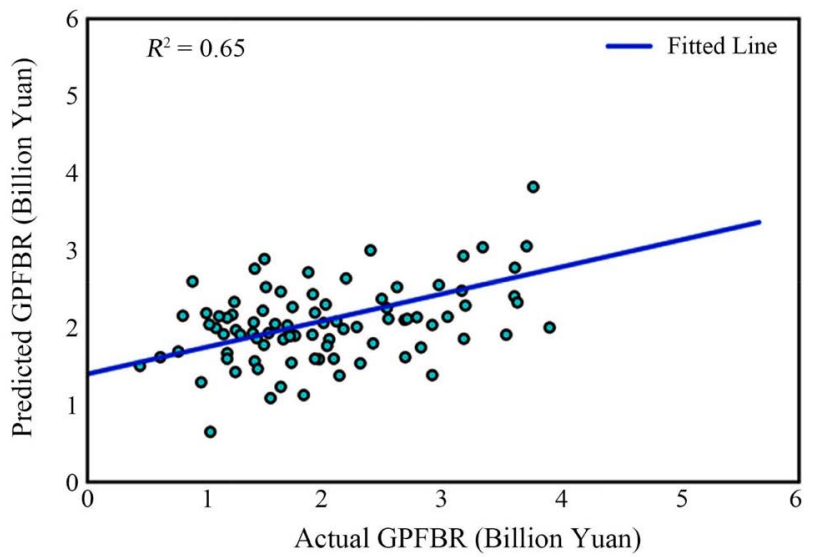

Figure 5. The Pearson coefficient $\left(\mathrm{R}^{2}\right)$ of the predicted GPFBR and the actual GPFBR, in which the blue line is the best fitting line.

Although this study shows that the combination of CNN and remote sensing image has a high accuracy in regional poverty estimation, it is still necessary to further study the applicability of deep learning in different regional poverty estimation. 


\section{Acknowledgements}

We would like to thank Neal Jean for publishing the code for the feature training ridge regression used to estimated economic indicators. Our work is implemented by Tensorflow and Keras API, and the data is collected by Google Earth Engine.

\section{Funding}

This work is financially supported by the Project for Follow-Up Work in Three Gorges (12610100000018J107).

\section{Conflicts of Interest}

The authors declare no conflicts of interest regarding the publication of this paper.

\section{References}

[1] Zhao, X., et al. (2019) Estimation of Poverty Using Random Forest Regression with Multi-Source Data: A Case Study in Bangladesh. Remote Sensing, 11, 375. https://doi.org/10.3390/rs11040375

[2] Engstrom, R., Newhouse, D., Haldavanekar, V., Copenhaver, A. and Hersh, J. (2017) Evaluating the Relationship between Spatial and Spectral Features Derived from High Spatial Resolution Satellite Data and Urban Poverty in Colombo, Sri Lanka. 2017 Joint Urban Remote Sensing Event, Dubai, 6-8 March 2017, 8-11. https://doi.org/10.1109/JURSE.2017.7924590

[3] Ferreira, F.H.G., et al. (2016) A Global Count of the Extreme Poor in 2012: Data Issues, Methodology and Initial Results. The Journal of Economic Inequality, 14, 141-172. https://doi.org/10.1007/s10888-016-9326-6

[4] Sutton, P.C. and Costanza, R. (2002) Global Estimates of Market and Non-Market Values Derived from Nighttime Satellite Imagery, Land Cover, and Ecosystem Service Valuation. Ecological Economics, 41, 509-527. https://doi.org/10.1016/S0921-8009(02)00097-6

[5] Doll, C.N.H., Muller, J.P. and Morley, J.G. (2006) Mapping Regional Economic Activity from Night-Time Light Satellite Imagery. Ecological Economics, 57, 75-92. https://doi.org/10.1016/j.ecolecon.2005.03.007

[6] Cui, X., Lei, Y., Zhang, F., Zhang, X. and Wu, F. (2019) Mapping Spatiotemporal Variations of $\mathrm{CO}_{2}$ (Carbon Dioxide) Emissions Using Nighttime Light Data in Guangdong Province. Physics and Chemistry of the Earth, 110, 89-98. https://doi.org/10.1016/j.pce.2019.01.007

[7] Ou, J., Liu, X., Li, X., Li, M. and Li, W. (2015) Evaluation of NPP-VIIRS Nighttime Light Data for Mapping Global Fossil Fuel Combustion $\mathrm{CO}_{2}$ Emissions: A Comparison with DMSP-OLS Nighttime Light Data. PLoS ONE, 10, e0138310. https://doi.org/10.1371/journal.pone.0138310

[8] Marx, A. and Ziegler Rogers, M. (2017) Analysis of Panamanian DMSP/OLS Nightlights Corroborates Suspicions of Inaccurate Fiscal Data: A Natural Experiment Examining the Accuracy of GDP Data. Remote Sensing Applications: Society and Environment, 8, 99-104, . https://doi.org/10.1016/j.rsase.2017.08.005

[9] Shi, K., et al. (2014) Evaluating the Ability of NPP-VIIRS Nighttime Light Data to 
Estimate the Gross Domestic Product and the Electric Power Consumption of China at Multiple Scales: A Comparison with DMSP-OLS Data. Remote Sensing, 6, 1705-1724. https://doi.org/10.3390/rs6021705

[10] Zhao, N., Liu, Y., Cao, G., Samson, E.L. and Zhang, J. (2017) Forecasting China's GDP at the Pixel Level Using Nighttime Lights Time Series and Population Images. GIScience \& Remote Sensing, 54, 407-425. https://doi.org/10.1080/15481603.2016.1276705

[11] Sutton, P., Roberts, D., Elvidge, C. and Baugh, K. (2001) Census from Heaven: An Estimate of the Global Human Population Using Night-Time Satellite Imagery. International Journal of Remote Sensing, 22, 3061-3076. https://doi.org/10.1080/01431160010007015

[12] Li, C., Li, G., Zhu, Y., Ge, Y., Te Kung, H. and Wu, Y. (2017) A Likelihood-Based Spatial Statistical Transformation Model (LBSSTM) of Regional Economic Development Using DMSP/OLS Time-Series Nighttime Light Imagery. Spatial Statistics, 21, 421-439. https://doi.org/10.1016/j.spasta.2017.03.004

[13] Wu, J., Wang, Z., Li, W. and Peng, J. (2013) Exploring Factors Affecting the Relationship between Light Consumption and GDP Based on DMSP/OLS Nighttime Satellite Imagery. Remote Sensing of Environment, 134, 111-119. https://doi.org/10.1016/j.rse.2013.03.001

[14] Elvidge, C.D., Zhizhin, M., Hsu, F.C. and Baugh, K.E. (2013) VIIRS Nightfire: Satellite Pyrometry at Night. Remote Sensing, 5, 4423-4449. https://doi.org/10.3390/rs5094423

[15] Yu, B., et al. (2014) Object-Based Spatial Cluster Analysis of Urban Landscape Pattern Using Nighttime Light Satellite Images: A Case Study of China. International Journal of Geographical Information Science, 28, 2328-2355. https://doi.org/10.1080/13658816.2014.922186

[16] Elvidge, C.D., Zhizhin, M., Hsu, F.-C. and Baugh, K. (2013) What Is So Great about Nighttime VIIRS Data for the Detection and Characterization of Combustion Sources? Proceedings of the Asia-Pacific Advanced Network, 35, 33-48. https://doi.org/10.7125/apan.35.5

[17] Elvidge, C.D., Baugh, K.E., Zhizhin, M. and Hsu, F.-C. (2013) Why VIIRS Data Are Superior to DMSP for Mapping Nighttime Lights. Proceedings of the Asia-Pacific Advanced Network, 35, 62-69. https://doi.org/10.7125/APAN.35.7

[18] Noor, A.M., Alegana, V.A., Gething, P.W., Tatem, A.J. and Snow, R.W. (2008) Using Remotely Sensed Night-Time Light as a Proxy for Poverty in Africa. Population Health Metrics, 6, 5. https://doi.org/10.1186/1478-7954-6-5

[19] Li, X., Xu, H., Chen, X. and Li, C. (2013) Potential of NPP-VIIRS Nighttime Light Imagery for Modeling the Regional Economy of China. Remote Sensing, 5, 3057-3081. https://doi.org/10.3390/rs5063057

[20] Yu, B., Shi, K., Hu, Y., Huang, C., Chen, Z. and Wu, J. (2015) Poverty Evaluation Using NPP-VIIRS Nighttime Light Composite Data at the County Level in China. IEEE Journal of Selected Topics in Applied Earth Observations and Remote Sensing, 8, 1217-1229. https://doi.org/10.1109/JSTARS.2015.2399416

[21] Jean, N., Burke, M., Xie, M., Davis, W.M., Lobell, D.B. and Ermon, S. (2016) Combining Satellite Imagery and Machine Learning to Predict Poverty. Science, 353, 790-794. https://doi.org/10.1126/science.aaf7894

[22] Perez, A., Ganguli, S., Ermon, S., Azzari, G., Burke, M. and Lobell, D. (2019) Semi-Supervised Multitask Learning on Multispectral Satellite Images Using Wasserstein Generative Adversarial Networks (GANs) for Predicting Poverty. 
[23] He, K., Zhang, X., Ren, S. and Sun, J. (2016) Deep Residual Learning for Image Recognition. 2016 IEEE Conference on Computer Vision and Pattern Recognition, Las Vegas, NV, 27-30 June 2016, 770-778. https://doi.org/10.1109/CVPR.2016.90

[24] Lin, T.Y., Dollár, P., Girshick, R., He, K., Hariharan, B. and Belongie, S. (2017) Feature Pyramid Networks for Object Detection. 2017 IEEE Conference on Computer Vision and Pattern Recognition, Honolulu, HI, 21-26 July 2017, 936-944. https://doi.org/10.1109/CVPR.2017.106

[25] Gallo, K.P., Owen, T.W., Gallo, K.P. and Owen, T.W. (1999) Satellite-Based Adjustments for the Urban Heat Island Temperature Bias. Journal of Applied Meteorology, 38, 806-813. https://doi.org/10.1175/1520-0450(1999)038<0806:SBAFTU>2.0.CO;2

[26] Yuan, F. and Bauer, M.E. (2007) Comparison of Impervious Surface Area and Normalized Difference Vegetation Index as Indicators of Surface Urban Heat Island Effects in Landsat Imagery. Remote Sensing of Environment, 106, 375-386. https://doi.org/10.1016/j.rse.2006.09.003

[27] Xu, H. (2006) Modification of Normalised Difference Water Index (NDWI) to Enhance Open Water Features in Remotely Sensed Imagery. International Journal of Remote Sensing, 27, 3025-3033. https://doi.org/10.1080/01431160600589179

[28] Zha, Y., Gao, J. and Ni, S. (2003) Use of Normalized Difference Built-up Index in Automatically Mapping Urban Areas from TM Imagery. International Journal of Remote Sensing, 24, 583-594. https://doi.org/10.1080/01431160304987

[29] Guizhou Provincial Bureau of Statistics. http://stjj.guizhou.gov.cn/tjsj_35719/sjcx_35720/gztjnj_40112/tjnj2018/

[30] Xie, M., Jean, N., Burke, M., Lobell, D. and Ermon, S. (2015) Transfer Learning from Deep Features for Remote Sensing and Poverty Mapping. Computer Science, 3929-3935. 